Article

Subscriber access provided by King Abdullah University of Science and Technology Library

\title{
Layer-Dependent Rashba Band Splitting in 2D Hybrid Perovskites
}

Jun Yin, Partha Maity, Liangjin Xu, Ahmed M. El-Zohry, Hong

Li, Osman M. Bakr, Jean-Luc Brédas, and Omar F. Mohammed

Chem. Mater., Just Accepted Manuscript • DOI: 10.1021/acs.chemmater.8b03436 • Publication Date (Web): 12 Oct 2018

Downloaded from http://pubs.acs.org on October 16, 2018

\section{Just Accepted}

"Just Accepted" manuscripts have been peer-reviewed and accepted for publication. They are posted online prior to technical editing, formatting for publication and author proofing. The American Chemical Society provides "Just Accepted" as a service to the research community to expedite the dissemination of scientific material as soon as possible after acceptance. "Just Accepted" manuscripts appear in full in PDF format accompanied by an HTML abstract. "Just Accepted" manuscripts have been fully peer reviewed, but should not be considered the official version of record. They are citable by the Digital Object Identifier (DOI®). "Just Accepted" is an optional service offered to authors. Therefore, the "Just Accepted" Web site may not include all articles that will be published in the journal. After a manuscript is technically edited and formatted, it will be removed from the "Just Accepted" Web site and published as an ASAP article. Note that technical editing may introduce minor changes to the manuscript text and/or graphics which could affect content, and all legal disclaimers and ethical guidelines that apply to the journal pertain. ACS cannot be held responsible for errors or consequences arising from the use of information contained in these "Just Accepted" manuscripts. 


\title{
Layer-Dependent Rashba Band Splitting in 2D Hybrid Perovskites
}

\author{
Jun Yin,${ }^{\dagger}$ Partha Maity,${ }^{\dagger}$ Liangjin Xu,${ }^{\dagger}$ Ahmed M. El-Zohry,${ }^{\dagger}$ Hong Li, ${ }^{\ddagger}$ Osman M. Bakr,${ }^{\dagger}$ \\ Jean-Luc Brédas, ${ }^{*}, \neq$ and Omar F. Mohammed ${ }^{*}, \dagger$ \\ † Division of Physical Science and Engineering, King Abdullah University of Science and Technology, Thuwal 23955- \\ 6900, Kingdom of Saudi Arabia. \\ ₹ School of Chemistry and Biochemistry, Center for Organic Photonics and Electronics (COPE), Georgia Institute of \\ Technology, Atlanta, Georgia 30332-0400, United States.
}

\begin{abstract}
The strong spin-orbit coupling (SOC) in perovskite materials due to the presence of heavy atoms induces interesting electronic characteristics, such as Rashba band splitting. In spite of several recent reports on Rashba effects in $2 \mathrm{D}$ perovskites, the impacts of the nature of surface termination and of the number of inorganic layers on the extent of Rashba band splitting still remain to be determined. Here, using a combination of density functional theory (DFT) calculations and time-resolved laser spectroscopy, we provide a comprehensive understanding of the Rashba band splitting of the prototype $3 \mathrm{D} \mathrm{MAPbI}_{3}$ and of $2 \mathrm{D}$ Ruddlesden-Popper (RP) hybrid perovskites. We demonstrate that significant structural distortions associated with different surface terminations are responsible for the observed Rashba effect in $2 \mathrm{D}$ perovskites. Interestingly, our theoretical and experimental data clearly indicate that the intrinsic Rashba splitting occurs in the perovskite crystals with an even number of inorganic layers $(n=2)$, but not for the ones with an odd number of layers $(n=1$ and $n=3)$. These findings not only provide a possible explanation for the elongated electron-hole recombination in perovskites but also elucidate the significant impact of the number of inorganic layers on the electronic properties of $2 \mathrm{D}$ perovskites.
\end{abstract}

\section{INTRODUCTION}

Hybrid organic-inorganic perovskites, such as methyl ammonium lead iodide $\left(\mathrm{MAPbI}_{3}\right)$, have recently attracted great attention due to several promising optoelectronic device applications, such as solar cells, ${ }^{1-5}$ light-emitting diodes, ${ }^{6-8}$ photodetectors, ${ }^{9-11}$ lasers, ${ }^{12}$ and scintillators. ${ }^{13}$ These hybrid materials exhibit strong spin-orbit coupling (SOC) due to the presence of heavy elements (i.e., $\mathrm{Pb}$ ), which gives rise to a large Rashba splitting induced by symmetry breaking. ${ }^{14-16}$ The Rashba effect provides an important pathway for electronic spin manipulation, which is one of the key components in realizing a semiconductor spintronic device. ${ }^{17-18}$ The original description of the Rashba effect goes back to the 1950's and corresponds to spin splitting in non-centrosymmetric semiconductors. ${ }^{19-20}$ More recently, Bychkov and Rashba noted that the effect also occurs in two-dimensional electron gas (2DEG) systems. ${ }^{21}$ A few years ago, the discovery of a giant Rashba splitting in the $3 \mathrm{D}$ material $\mathrm{BiTeI}^{22}$ inspired spintronic studies involving some ${ }_{3} \mathrm{D}$ and two-dimensional (2D) hybrid perovskites ${ }^{23-25}$. In these novel ferroelectric Rashba materials, the ferroelectric polarization can directly couple to the spin splitting and the helical spin texture once the inversion symmetrybreaking field is generated; this enables effective control and manipulation of the polarized spins in spintronic devices. Furthermore, the broad tunability of bandgap and SOC in perovskite materials through chemical substitutions provides extra flexibility in device design. These advantages, together with their optical accessibility for spin generation and detection, make hybrid perovskites attractive for spintronic applications. ${ }^{26}$
In hybrid perovskites, spin splitting can contribute strongly to the direct-indirect character of bandgap, ${ }^{27-28}$ slow charge carrier dynamics, ${ }^{29-31}$ unusual magneto-optical properties $^{32}$, and the bright triplet emissions. ${ }^{3}$ Experimentally, Hsiao et al. have demonstrated that the photogenerated charge carriers undergo a spin-dependent electron-hole recombination and charge dissociation in $\mathrm{MAPbI}_{3} \cdot{ }^{34}$ On the other hand, a large Rashba splitting of the valence bands in $\mathrm{MAPbBr}_{3}$ single crystals has been reported by Fauster and co-workers. 35 In that work, the possibility to create spin polarization in these hybrid perovskites was demonstrated, making them indeed excellent candidates for new applications, such as in spintronic devices. ${ }^{6}$ On the theoretical side, a Rashba spin splitting has been described in $3 \mathrm{D}$ hybrid perovskites, such as $\operatorname{MAPbX}_{3}(\mathrm{X}=\mathrm{Br}$ and $\mathrm{I})$ and $\mathrm{FASnI}_{3}{ }^{15}$, 37 However, since spin splitting is extremely sensitive to structural optimizations, the impact of distortions of the inorganic framework on the actual extent of Rashba splitting in low dimensional hybrid perovskites remain unclear, especially with regard to the characterization of the spin-polarized states and the impact of the inorganic number of layers in the $2 \mathrm{D}$ Ruddlesden-Popper (RP) perovskite structures.

In this Article, we explore the Rashba effect in a series of hybrid perovskites based on the analysis of crystal structure and band splitting using DFT calculations and ultrafast time-resolved spectroscopy. We first investigate, for the sake of reference, the general features of Rashba spin splitting in prototype $3 \mathrm{D}$ hybrid perovskites using different DFT methodologies. Then, we treat the $2 \mathrm{D}$ form of $\mathrm{MAPbI}_{3}$ and consider different terminations, in which bulk lattice and surface structural distortions contribute to the strong Rashba band splitting; here, we use the PBE 
functional method with SOC and van der Waals interactions. Finally, we study stable $2 \mathrm{D}$ RuddlesdenPopper hybrid perovskites with both centrosymmetric $(n=$ 1 and 3 ) and non-centrosymmetric $(n=2)$ crystal structures and demonstrate how the band splitting slows down the charge-carrier recombination in the case of $n=2$.

\section{EXPERIMENTAL DETAILS}

Computational Methods. Density functional theory (DFT) calculations were performed to optimize the crystal structures of ${ }_{3} \mathrm{D}$ and $2 \mathrm{D}$ forms of $\mathrm{MAPbI}_{3}$ and $2 \mathrm{D} \mathrm{RP}$ hybrid perovskites using the projector augmented wave (PAW) method as implemented in the VASP code. ${ }^{8-39}$ In all instances, the experimental crystal structures at room temperature were used as starting points and further optimized by relaxing both crystal lattices and atomic positions. To compare the electronic bands of bulk $\mathrm{MAPbI}_{3}$ using different DFT methodologies, the PBE functional was used with and without consideration of SOC and $\mathrm{vdW}$ effects. The plane-wave cutoff energy was set to $500.0 \mathrm{eV}$ as the well convergence of total energy was obtained (see Figure $S_{1}$ of the Supporting Information). Note that the width of Gaussian smearing was set to $0.05 \mathrm{eV}$; selfconsistent field (SCF) energy convergence critical was set to $1 \times 10^{-5} \mathrm{eV}$; and $\Gamma$-centered $6 \times 6 \times 4$ and $6 \times 6 \times 1 \mathrm{k}$-meshes in the Brillouin zone were employed for bulk $\mathrm{MAPbI}_{3}$ and $2 \mathrm{D}$ RP hybrid perovskites, respectively. The experimental and optimized crystal parameters and atomic coordinates of $3 \mathrm{D}$ $\mathrm{MAPbI}_{3}$ and $2 \mathrm{D}$ RP hybrid perovskites are given in Tables $\mathrm{S}_{1}, \mathrm{~S}_{2}$ and $\mathrm{S}_{5}$-S8 of the Supporting Information. All slabs exposed the $\mathrm{MAPbI}_{3}$ (oo1) surface with different terminations and a $6 \times 6 \times 1 \mathrm{k}$-mesh was used. All slabs were separated by both top and bottom vacuum layers $(\sim 15 \AA)$ to prevent spurious inter-slab interactions; the slab structures were further optimized under different external electric fields until the total force on each atom was $<0.01$ $\mathrm{eV} / \mathrm{A}$. The molecular graphics viewer (VESTA) was used to plot all the bulk and slab structures.

Single-Crystal Synthesis. All reagents were used without any purification: hydriodic acid $(57 \% \mathrm{w} / \mathrm{w}$ in water, Alpha Aesar), methylammonium iodide (>99\%, Dyesol) and lead oxide (99\%, Sigma-Aldrich). Methylammonium iodide (MAI), lead oxide $(\mathrm{PbO})$, and phenethylamine (PEA), in specific ratios, were dissolved in hydriodic acid (HI) solution in a $20 \mathrm{~mL}$ vial. Then, the vial was kept in an oven at $110^{\circ} \mathrm{C}$ for 4 hours. The $(\mathrm{PEA})_{2}(\mathrm{MA})_{\mathrm{n}-}$ ${ }_{1} \mathrm{~Pb}_{n} \mathrm{I}_{3 n+1}(n=1,2,3)$ single crystals were formed once the solution cooled to room temperature. The ratios of $\mathrm{PbO} / \mathrm{MAI} / \mathrm{PEA}$ are $1.72 / \mathrm{o} / 3.45 \mathrm{mmol}(n=1), 6 / 18 / 1 \mathrm{mmol}(n$ $=2)$, and $10 / 24 / 1 \mathrm{mmol}(n=3)$ in $30 \mathrm{~mL}$ of HI solution. More details are available in Ref ${ }^{40}$.

Time-Correlated Single Photon Counting. The 2D RP perovskite crystals were excited by a pulsed laser diode $(405 \mathrm{~nm})$ that was purchased from HORIBA Jobin Yvon, model (DD-405L, IRF $\approx 200 \mathrm{ps}$ ). The repetition rate of the pulsed laser ranges from a few $\mathrm{kHz}$ to $\mathrm{MHz}$ by a $\mathrm{DDC}_{1}$ picosecond controller also purchased from HORIBA. The laser energy per pulse used was approximately $1 \mathrm{pJ}$ (an excitation fluence of $0.05 \mu \mathrm{J} / \mathrm{cm}^{2}$ ). The laser light was tightly focused on the sample using an objective lens from Olympus Ltd. (Plan N, 10x/o.25). The maximized emission is collected from the same excited area by the objective lens after passing through a reflective beam splitter ( $R$ 488) from Thorlabs. Then, the collected emission was focused on a commercial Avalanche photodiode controlled by a Hydra Harp 400 multichannel picosecond event timer unit from PicoQuant to detect the emission in a single photon fashion, i.e., one per laser pulse at max. For the $n=1$ crystal, an additional green bandpass filter was used to block emissions coming from long-wavelength radiation at approximately $750 \mathrm{~nm}$.

Femtosecond Transient Absorption Spectroscopy. Femtosecond transient absorption (fs-TA) spectroscopy measurements were performed on timescales of o.1 ps to 6 $\mathrm{ns}$, with a regenerative amplified Ti:sapphire laser (80o $\mathrm{nm}$ laser pulses of $<120$ fs pulse width with a $1 \mathrm{kHz}$ repetition rate) and in conjunction with Helios spectrometers (Ultrafast Systems). Excitation pump pulses of 475, 520, and $580 \mathrm{~nm}$ were generated after passing through a fraction of the $800 \mathrm{~nm}$ beam into the spectrally tunable (240-2600 nm) optical parametric amplifier (Newport Spectra-Physics). The pump fluence of the excitation laser source was adjusted by using neutral density (ND) filters to avoid Auger recombination due to multiphoton processes. The probe pulses (UV visible and NIR wavelength continuum, white light) were generated by passing another fraction of the $800 \mathrm{~nm}$ pulses through the 2-mm thick sapphire crystal. Before white light generation, the $800 \mathrm{~nm}$ amplified pulses were passed through a motorized delay stage. Depending on the movement of delay stage, the transient species were detected following excitation at different time scales. The white light was split into two beams (named signal and reference) and focused on two fiber optics for improvement of the signal-to-noise ratio. The excitation pump pulses were spatially overlapped with the probe pulses on the samples after passing through a synchronized mechanical chopper (500 $\mathrm{Hz}$ ), which blocked alternative pump pulses. The reflected pulses were measured with respect to the time delay and wavelength. All spectra were averaged over $2 \mathrm{~s}$ for each time delay.

\section{RESULTS AND DISCUSSION}

Rashba splitting in ${ }_{3} \mathrm{D} \mathrm{MAPbI}_{3}$. The band dispersion relationships near the band edges (valence band maximum, VBM, and conduction band minimum, CBM) can be described by the effective mass approximation: The holes and electrons can be treated as charge carriers with a certain effective mass, related to the spin-degenerate parabolic dispersion. The SOC effect can split the (spindegenerate) parabolic band into two spin-polarized bands. In this case, the band dispersion relationship can be described by $E^{ \pm}(k)=\left(\hbar^{2} k^{2} / 2 m^{*}\right) \pm \alpha_{R}|k|$, where $\alpha_{R}$ is the Rashba splitting coefficient defined as $\alpha_{R}=2 E_{\mathrm{R}} / k_{\mathrm{o}}\left(k_{\mathrm{o}}\right.$ is the momentum offset, and $E_{R}$ is the energy splitting, as depicted in Figure $1 b)$. In the frame of this theoretical picture, we tested the effect in the prototype ferroelectric hybrid perovskite $\mathrm{MAPbI}_{3}$, since it has polar distortions 
(a)
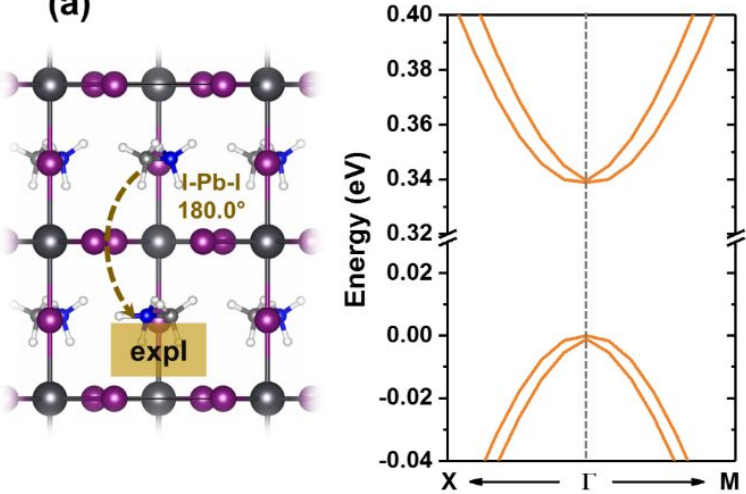

(b)

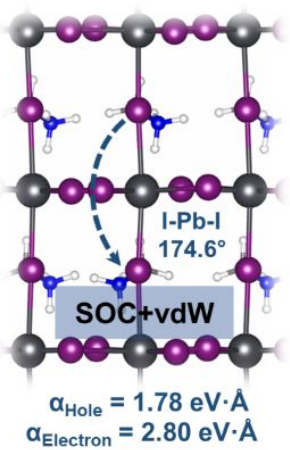

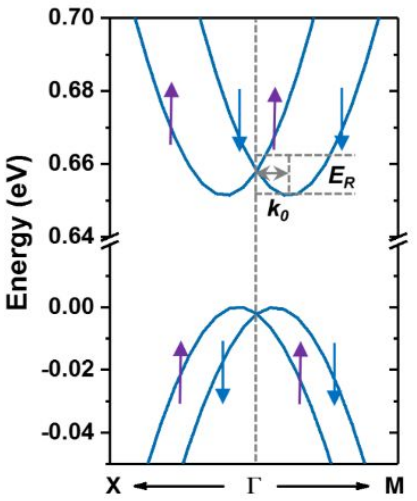

(c)

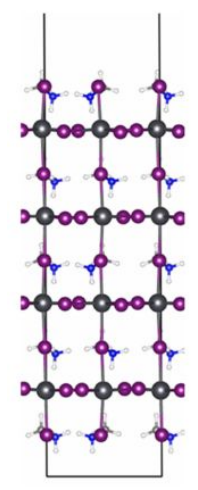

MAl-Terminated $\mathrm{MAPbl}_{3}$

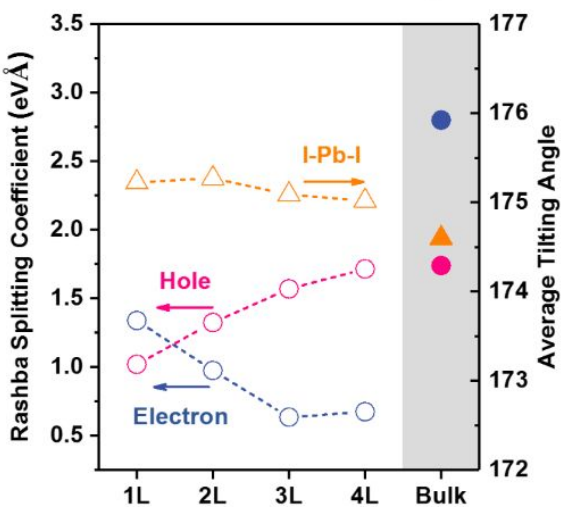

(d)

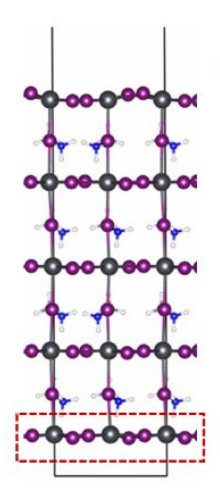

\section{$\mathrm{Pbl}_{2}$-Terminated $\mathrm{MAPbl}_{3}$}

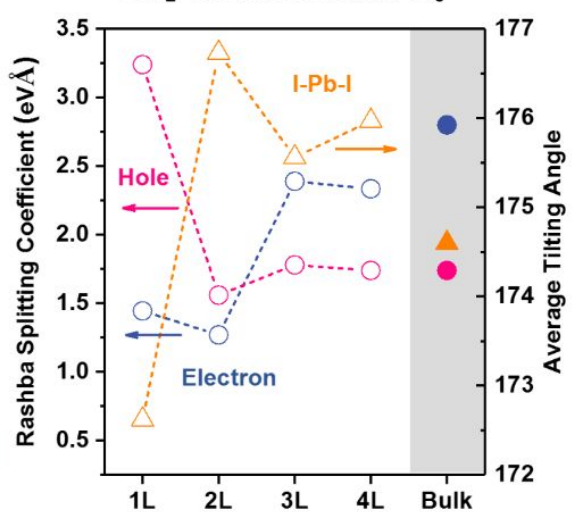

Figure 1. (a, b) Experimental and optimized crystal structures of tetragonal-phase $\mathrm{MAPbI}_{3}$ (with the indication of the I-Pb-I tilting angles) and corresponding electronic band structures calculated at the PBE+SOC+vdW level of theory; (c, d) optimized four-layer $(4 \mathrm{~L})$ slab structure, out-of-plane I-Pb-I tilting angles and calculated Rashba splitting coefficients for $1 \mathrm{~L}-4 \mathrm{~L}$ MAPbI ${ }_{3}$ slabs with both MAI- and $\mathrm{PbI}_{2}$-terminations.

with a significant ferroelectric response at room temperature. ${ }^{41}$ We first compared the optimized crystal parameters of tetragonal-phase $\mathrm{MAPbI}_{3}$ and corresponding Rashba coefficients $\left(\alpha_{R}\right)$ at different levels of DFT methodology. As illustrated in Tables $S_{1}$ and $S_{3}$ and Figure S2 of the Supporting Information, we find that: $i$ ) including van der Waals (vdW) interactions leads to more distorted crystal structures but slightly underestimates the $a$ and $b$ crystal parameters compared to the experimental values; and ii) combining SOC and vdW effects gives nearly the same values of Rashba coefficients along the $X \rightarrow \Gamma$ and $\Gamma \rightarrow M$ directions. Since including SOC effects and vdW interactions captures the heavy-atom effects as well as the non-covalent interactions in the crystal structure optimizations, we decided to use this methodology for all the following investigations.

As shown in Figure 1a and $1 \mathrm{~b}$, the experimental structure with space group of $14 / \mathrm{mcm}$ was also considered. The octahedron distortion was found to be negligible, i.e., the $\mathrm{I}-\mathrm{Pb}-\mathrm{I}$ angle was $180^{\circ}$. Once the crystal lattice and atomic positions were fully relaxed without keeping centrosymmetric Pb-I framework at the PBE+SOC+vdW level, a small polar distortion occurred along the $z$ direction, resulting in an off-centering of the $\mathrm{Pb}$ atom and reducing the $\mathrm{I}-\mathrm{Pb}-\mathrm{I}$ angle to $174.6^{\circ}$. It should be noted that the difference of lattice distortions between experimental and optimized crystal structure is not affected by DFT functional, such as PBEsol (see Table S1 of the Supporting Information). The calculated electronic band structures of $\mathrm{MAPbI}_{3}$ based on these two crystal structures are also shown in Fig $1(\mathrm{a}, \mathrm{b})$. As expected, the experimental symmetrical crystal structure results in almost no band splitting; in contrast, for the fully optimized crystal, the VBM and CBM near the $\Gamma$-point indicate a large Rashbatype band splitting; the estimated Rashba coefficients for the hole and electron in the optimized $\mathrm{MAPbI}_{3}$ structure are $1.78 \mathrm{eV} \cdot \AA$ and $2.8 \mathrm{o} \mathrm{eV} \cdot \AA$, respectively. Thus, the CBM, which is dominated by $\mathrm{Pb}$ atoms, exhibits a significantly larger splitting coefficient than the VBM. Our calculated Rashba coefficients for bulk $\mathrm{MAPbI}_{3}$ are on the same order of magnitude as those reported earlier (1.5-3.7 eV·Å).15, 37, 42 We note that the $\mathrm{MAPbX}_{3}$ systems are promising hybrid materials for spintronic applications. Large $\alpha_{R}$ values $(7 \pm 1$ and $11 \pm 4 \mathrm{eV} \cdot \AA$ for the orthorhombic and cubic phases, respectively) were experimentally observed in $\mathrm{MAPbBr}_{3} ; 35$ moreover, the Rashba coefficients in hybrid $\mathrm{MAPbX}_{3}$ systems are much larger than their inorganic perovskite counterparts $43\left(\mathrm{CsPbr}_{3}, 0.2 \mathrm{eV} \cdot \AA\right.$ for electrons and 0.5 $\mathrm{eV} \cdot \AA$ for holes) due to crystal symmetry-breaking induced by hydrogen bonding interactions between organic cations and inorganic framework, and these values are comparable to other bulk material systems, such as ultrathin metallic 
films (Bi/Ag, $3.05 \mathrm{eV} \cdot \AA$ A $),{ }^{44}$ topological insulators $\left(\mathrm{Bi}_{2} \mathrm{Se}_{3}\right.$, (a)

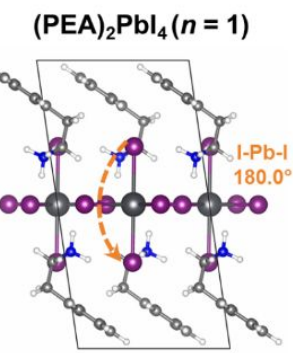

(b)

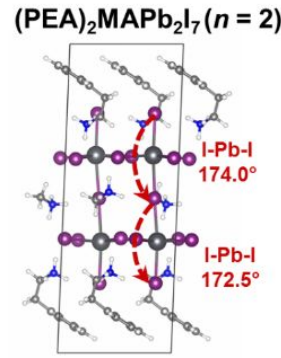

(c)

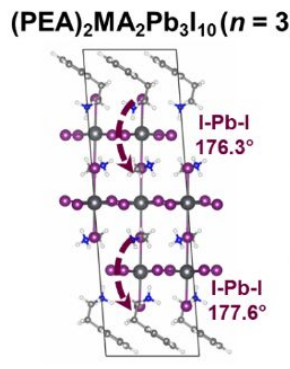

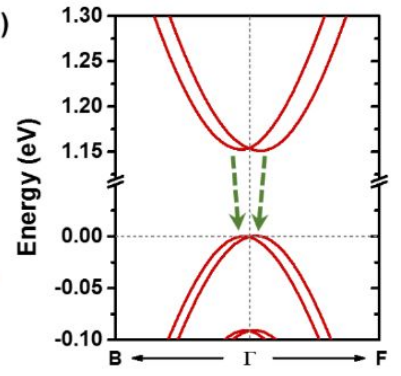
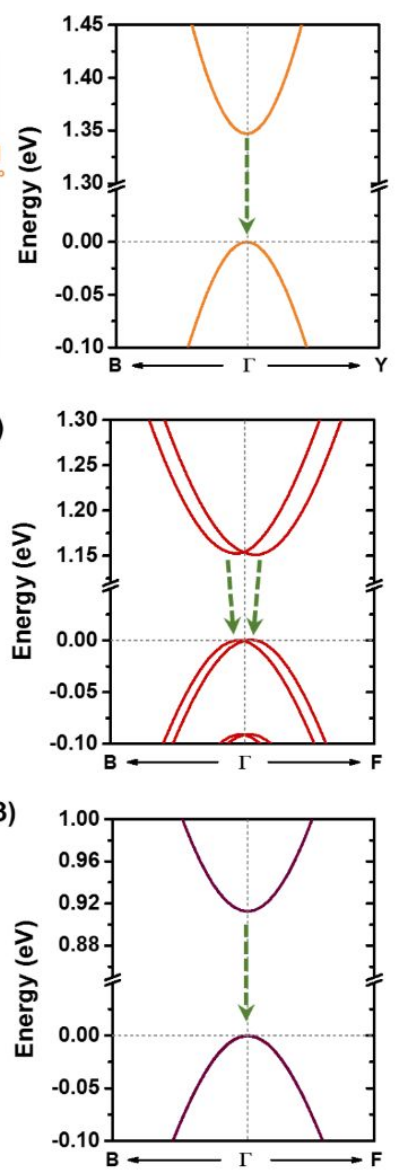

Figure 2. (a) Optimized crystal structures (with indication of the I-Pb-I tilting angles) and electronic band structures of (a) (PEA) ${ }_{2} \mathrm{PbI}_{4}(n=1)$, (b) (PEA) ${ }_{2} \mathrm{MAPb}_{2} \mathrm{I}_{7}(n$ $=2)$, and (c) $(\mathrm{PEA})_{2} \mathrm{MA}_{2} \mathrm{~Pb}_{3} \mathrm{I}_{10}(n=3)$ calculated at the $\mathrm{PBE}+\mathrm{SOC}+\mathrm{vdW}$ level of theory.

$3.20 \mathrm{eV} \cdot \AA$ ), 45 and polar semiconductors (BiTeI, $3.8 \mathrm{o} \mathrm{eV} \cdot \AA$ ). ${ }^{22}$

Rashba splitting in 2D layered MAPbI. After describing the presence of Rashba splitting in bulk materials, we used the same methodology to explore the effects of the number of layers and surface termination on the band splitting feature in $2 \mathrm{D} \mathrm{MAPbI}{ }_{3}$ (oor) slabs. The band structures of optimized slabs calculated at the $\mathrm{PBE}+\mathrm{SOC}+\mathrm{vdW}$ level are shown in Figure $\mathrm{S}_{3}$ and $\mathrm{S}_{4}$ of the Supporting Information. The out-of-plane tilting angles and the deduced $\alpha_{\mathrm{R}}$ values for MAI- and $\mathrm{PbI}_{2}$-terminated slabs are shown in Figure ic and id for the sake of comparison. We find that all $\mathrm{MAPbI}_{3}$ (ool) slabs show Rashba splitting characteristics, but overall they have smaller $\alpha_{R}$ values than those in the bulk. For MAIterminated slabs, the $\alpha_{R}$ values for holes increase when increasing the number of layers from ${ }_{1} \mathrm{~L}$ to $4 \mathrm{~L}$; on the other hand, the $\alpha_{\mathrm{R}}$ values for electrons decrease from $1 \mathrm{~L}$ to ${ }_{3} \mathrm{~L}$ and then slightly increase from ${ }_{3} \mathrm{~L}$ to $4 \mathrm{~L}$. In the case of $\mathrm{PbI}_{2}-$ terminated slabs, $\alpha_{R}$ values for holes and electrons show the same trend when increasing the number of layers: they increase from $2 \mathrm{~L}$ to $3 \mathrm{~L}$ and then slightly decrease from $3 \mathrm{~L}$ to $4 \mathrm{~L}$. Interestingly, for both terminations, the $\alpha_{\mathrm{R}}$ values for holes are strongly correlated with the out-of-plane I-Pb-I angles (i.e., the larger the tilting of the I-Pb-I angles, the larger the $\alpha_{\mathrm{R}}$ values). In addition, both ${ }_{3} \mathrm{~L}$ and ${ }_{4}{\mathrm{~L} \mathrm{PbI}_{2}-}^{-}$ terminated slabs have much larger $\alpha_{R}$ values for electrons as compared to the MAI-terminated ones; also, a very large

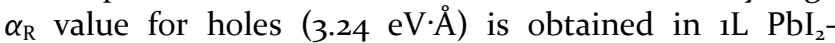
terminated slab. This can be ascribed to additional surface in-plane structural distortion induced by the unsaturated $\mathrm{Pb}$ atom (highlighted by the dashed boxes in Figure $1 d$, left) in the $\mathrm{PbI}_{2}$-terminated slabs. Therefore, the observed Rashba effect in $2 \mathrm{D} \mathrm{MAPbI}_{3}$ slabs are strongly dependent on the number of inorganic layers (related to inner-layer out-of-plane distortions) and the surface terminations (related to surface in-plane structural distortions).

Rashba splitting in $2 \mathrm{D}$ (PEA) 2 (MA) $)_{n-1} \mathrm{~Pb}_{\mathbf{n}} \mathrm{I}_{3 \mathrm{n}+1}$. Although $2 \mathrm{D} \mathrm{MAPbI}_{3}$ slab structures containing a few layers are calculated to have relatively large Rashba splitting coefficients, such slab structures are not stable as they are sensitive to humidity during crystal growth. One strategy to overcome the degradation issue is surface treatments: for example, Lewis bases (e.g., pyridine) have been used to terminate and stabilize the $\mathrm{PbBr}_{2}$-rich $\mathrm{MAPbBr}_{3}$ surface ${ }^{46}$, while long organic cations (e.g., phenylethylammonium, PEA) have been used to separate MAI-rich slabs and form stable $2 \mathrm{D}$ RP hybrid perovskites. In the latter case, the resulting single-crystal structures of $(\mathrm{PEA})_{2} \mathrm{PbI}_{4}(n=1), \quad(\mathrm{PEA})_{2} \mathrm{MAPb}_{2} \mathrm{I}_{7}(n=2)$, and $(\mathrm{PEA})_{2} \mathrm{MA}_{2} \mathrm{~Pb}_{3} \mathrm{I}_{10} \quad(n=3)$, have been experimentally confirmed in our previous work..$^{\circ}$ Here, we examine the Rashba splitting features of these $2 \mathrm{D}$ RP perovskite systems in order to assess whether these stable hybrid perovskites can have large $\alpha_{R}$ values appropriate for spintronic applications. As shown in Figure 2, the long organic PEA cations are rigid and can lock the crystal structure in preferred orientations, eliminating the tilting of the I-Pb-I angles.

From the calculated electronic band structures based on the optimized crystal structures, we find that an even number of layers $(n=2)$ belongs to the noncentrosymmetric group $C_{2} / m$ with splitting coefficients $\alpha_{R}$ $=1.08[0.67] \mathrm{eV} \cdot \AA$ A for electrons [holes] along $\Gamma \rightarrow B$ and $\alpha_{R}=$ 1.32 [1.01] eV·Å along $\Gamma \rightarrow F$. In contrast, an odd number of layers $(n=1$ and $n=3)$ leads to a centrosymmetric $P \overline{1}$ space group, and no splitting is observed for either CBM or VBM. Note that the bands along the $\Gamma \rightarrow Z$ direction (i.e., vertical to the inorganic layer) are flat without any splitting. Therefore, the impact of octahedron distortions induced by in-plane and out-of-plane tiltings illustrate the delicate symmetry/asymmetry balance required to observe a Rashba effect in this class of 2D RP hybrid materials.

Photoluminescence Lifetime. To experimentally confirm the band splitting in (PEA) ${ }_{2} \mathrm{MAPb}_{2} \mathrm{I}_{7}(n=2)$, we synthesized 2D RP hybrid perovskite single crystals with different numbers of layers using a modified synthetic protocol (see Experimental Methods). The as-grown single crystals display orthorhombic $2 \mathrm{D}$ RP perovskite crystal structures confirmed by X-ray diffraction (XRD) patterns (see Figure $\mathrm{S}_{5}$, Supporting Information). As shown in Figure 3a, these $2 \mathrm{D}$ hybrid $\mathrm{RP}$ perovskite single crystals exhibit tunable emission wavelengths with peaks at $527 \mathrm{~nm}$ 

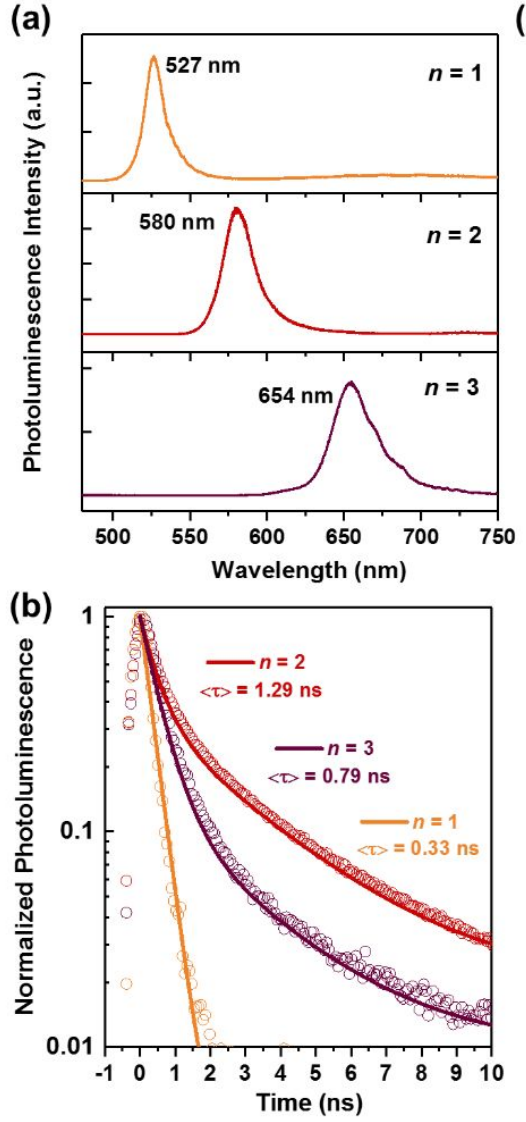

(c)
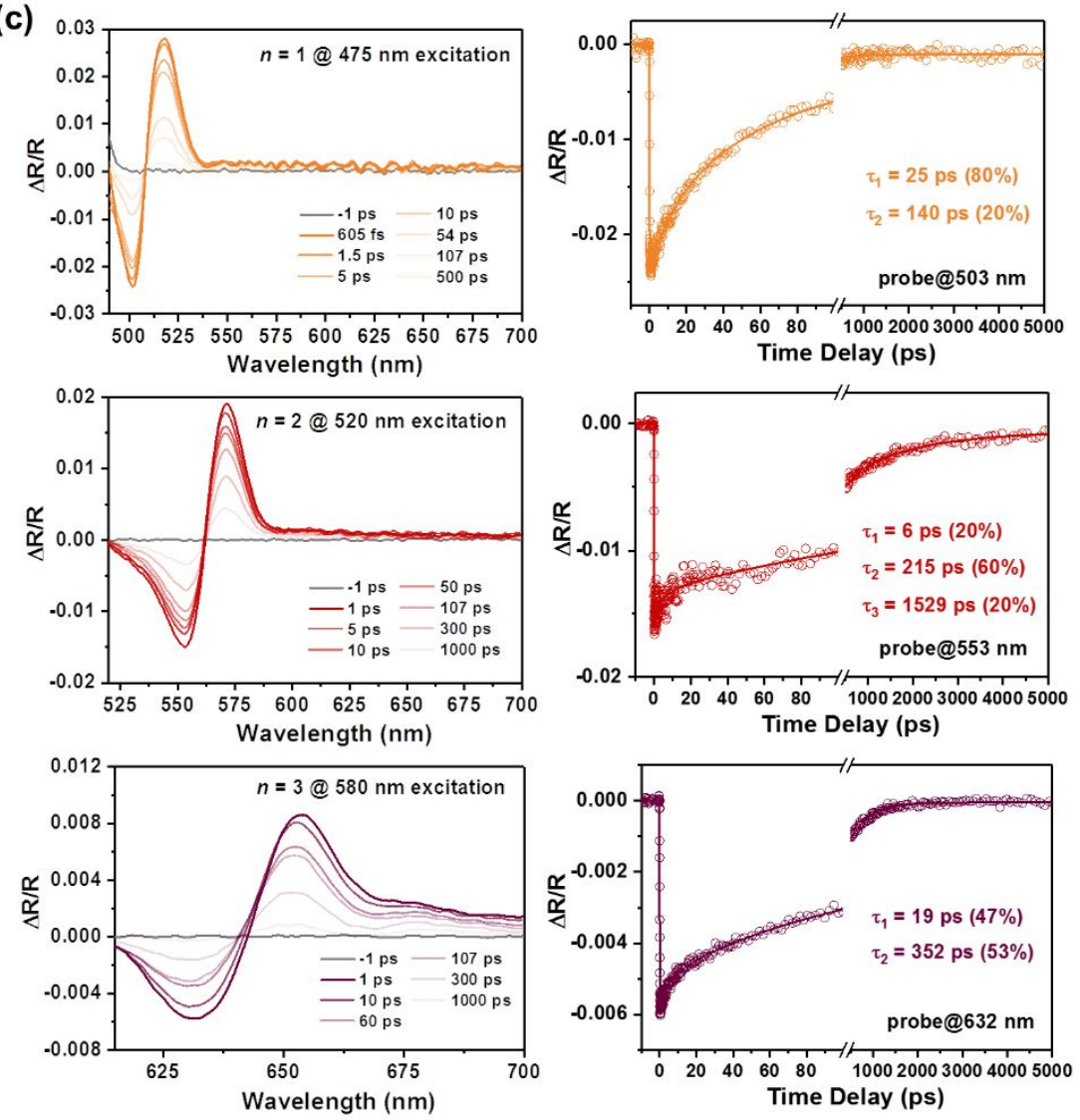

Figure 3. (a) Steady-state photoluminescence spectra; (b) Time-resolved photoluminescence spectra (excitation wavelength $=405 \mathrm{~nm}$ and power fluence $=0.05 \mu \mathrm{J} / \mathrm{cm}^{2}$ ) and average PL lifetimes with a monoexponential fitting line for $n=1$ and biexponential fitting lines for $n=2$ and $n=3$ (the fitting parameters are given in Table $\mathrm{S}_{4}$ of the Supporting Information); and (c) Transient reflectance spectra using band-edge excitations and corresponding kinetics for the ground-state bleaching (GSB) peaks of $(\mathrm{PEA})_{2} \mathrm{PbI}_{4}(n=1),(\mathrm{PEA})_{2} \mathrm{MAPb}_{2} \mathrm{I}_{7}(n=2)$, and $(\mathrm{PEA})_{2} \mathrm{MA}_{2} \mathrm{~Pb}_{3} \mathrm{I}_{10}(n=3)$, with an excitation fluence of $4.76 \mu \mathrm{J} / \mathrm{cm}^{2}$ for $n=1,5.24 \mu \mathrm{J} / \mathrm{cm}^{2}$ for $n=2$, and $5.10 \mu \mathrm{J} / \mathrm{cm}^{2}$ for $n=3$.

$(2.35 \mathrm{eV}, n=1), 580 \mathrm{~nm}(2.14 \mathrm{eV}, n=2)$, and $654 \mathrm{~nm}(1.90$ $\mathrm{eV}, n=3)$, which agree well with the calculated bandgaps of $2.27 \mathrm{eV}(n=1), 2.17 \mathrm{eV}(n=2)$, and $1.94 \mathrm{eV}(n=3)$ at PBE level of theory and previous studies. ${ }^{40}$ Time-resolved photoluminescence (TRPL) measurements were performed to better understand the band splitting effect on the charge carrier dynamics of these $2 \mathrm{D}$ RP perovskites. Due to the electronic energy level offset and dielectric constant mismatch between the inorganic layers and the organic cations, quantum wells form within the inorganic layer and both electrons and holes are confined to the inorganic layer after photoexcitation. Therefore, the PL lifetimes of the $2 \mathrm{D}$ RP perovskites should decrease when decreasing the number of layers due to the quantum confinement effects that tend to enhance the charge carrier recombination rates. ${ }^{47}$ As a result (see Figure $3 \mathrm{~b}$ ), the $n=1$ crystal has the shortest PL lifetime (o.33 ns) while the $n=3$ crystal shows a much longer lifetime of $\langle\tau\rangle=0.79$ ns; this is due to the reduced quantum confinement effect (i.e., exciton wavefunction delocalization) and the enhancement of elastic electron-phonon scattering, which acts to suppress the charge recombination for the $n=3$ crystal. ${ }^{48}$ Interestingly, the $n=2$ crystal exhibits an unusual longer PL lifetime, $<\tau>=1.29 \mathrm{~ns}$, compared to the other two crystals. According to our calculated electronic band structures in Figure 2, in both $n=1$ and $n=3$ crystals, we can expect that the electrons in CBM recombines rapidly with holes in VBM through direct (radiative) recombination; however, in $n=2$ crystal, the excited electrons can decay from two split CBs and undergo slow thermally activated indirect recombination with holes in the split VBs.

Transient Absorption Spectra and Kinetics. To further confirm that a Rashba splitting appears in the $\mathrm{n}=2$ crystal, we carried out femtosecond pump-probe experiments on the three single crystals using band-edge excitations $[475 \mathrm{~nm}(\mathrm{n}=1), 520 \mathrm{~nm}(\mathrm{n}=2)$, and $580 \mathrm{~nm}(\mathrm{n}$ = 3)] and collected the relative differential signals in reflectance geometry $(\Delta R / R) .{ }^{49}$ As shown in Figure $3 c$, the transient reflectance (TR) spectra of the $n=1$ crystal exhibit negative features below $510 \mathrm{~nm}$, which can be assigned to ground-state bleaching (GSB) due to filling of the excitonic states, and a positive feature with a peak centered at 518 $\mathrm{nm}$, which is attributed to excited-state absorption (ESA) of the photogenerated charged species. For $n>1$ crystals, the TR spectra also consist of two main features: a negative GSB band centered at $553 \mathrm{~nm}(n=2)$ and $630 \mathrm{~nm}(n=3)$ and a positive ESA band centered at $571 \mathrm{~nm}(n=2)$ and 650 
(a)
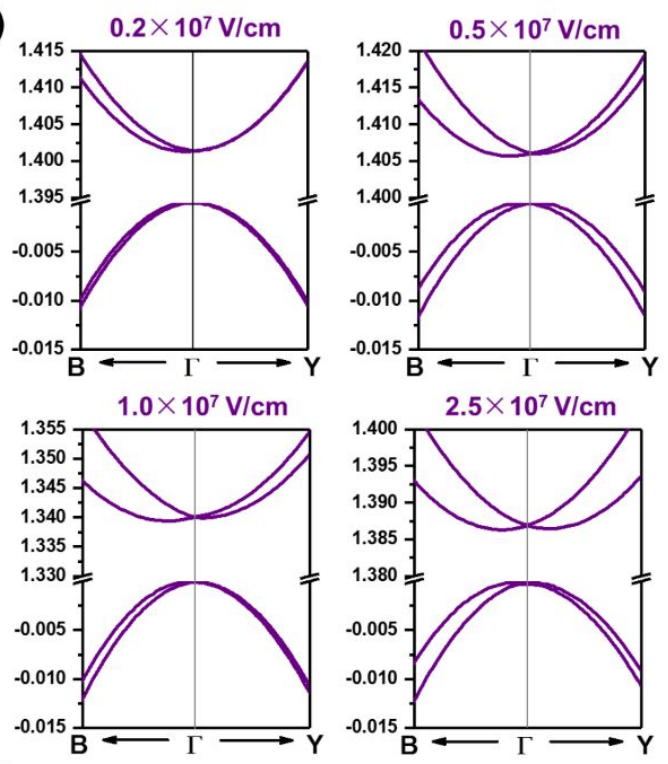

(b)

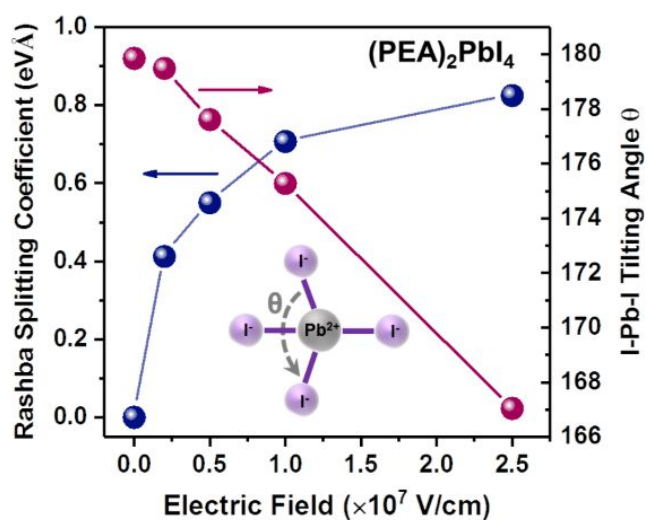

Figure 4. (a) Electronic VBM and CBM structures; and (b) Rashba splitting coefficients for electrons and I-Pb-I tilting angles for the $(\mathrm{PEA})_{2} \mathrm{PbI}_{4}$ slab calculated at $\mathrm{PBE}+\mathrm{SOC}+\mathrm{vdW}$ level as a function of an external electric field of $0.2 \times 10^{7}$ to $2.5 \times 10^{7} \mathrm{~V} / \mathrm{cm}$. The insets show the optimized (PEA) ${ }_{2} \mathrm{PbI}_{4}$ slab structures without and with an electric field of $2.5 \times 10^{7} \mathrm{~V} / \mathrm{cm}$.

$\mathrm{nm}(n=3)$. However, the kinetics of the transient reflectance peaks fitted with multiexponential functions show different decay lifetimes in these crystals. For the $n=$ 1 crystal, the TR kinetic probed at the GSB peak shows a fast decay with a lifetime constant of $\tau_{1}=25 \mathrm{ps}(8 \mathrm{o} \%)$ and $\tau_{2}=140 \mathrm{ps}(20 \%)$; the $n=3$ crystal has a slightly increased lifetime of $\tau_{1}=19 \mathrm{ps}(47 \%)$ and $\tau_{2}=352 \mathrm{ps}(53 \%)$. Interestingly, for the $n=2$ crystal, a fast decay kinetics is found for the GSB signal with a lifetime constant of $\tau_{1}=6$ ps $(20 \%)$ and $\tau_{2}=215$ ps $(60 \%)$, followed by a slow decay with a time constant of $\tau_{3}=1529$ ps $(20 \%)$. The trend of longer components in pump-probe measurements (i.e., 140,1529 , and 352 ps for $n=1,2$, and 3) agrees well with lifetimes obtained from TRPL measurements, which can be assigned to the band edge electron-hole recombination; while the short components (in sub-ps time scale) of these crystals could be attributed to the surface-trap states. Thus, the kinetics of both GSB and ESA for the $n=2$ crystal show significantly longer lifetimes in comparison with the other two crystals. According to our calculated electronic bands, in addition to the quantum confinement in the $n=$ 2 crystal, Rahsba band splitting plays a dominant role in achieving a long-lived GSB recovery signal.

Effects of Electric fields on Rashba splitting. One effective approach to achieve Rashba splitting in the $n=1$ and $n=3$ crystals is to apply a high external electric field that contributes to break the crystal symmetry. ${ }^{37,50}$ Recent experimental measurements show that a giant Rashba splitting coefficient value was achieved in a (PEA) ${ }_{2} \mathrm{PbI}_{4}$ 100$\mathrm{nm}$ film $(1.6 \pm 0.1 \mathrm{eV} \cdot \AA \AA$ ) under a high voltage of $50-325 \mathrm{~V}$ (i.e., electric field $=0.5 \times 10^{7}-3.25 \times 10^{7} \mathrm{~V} / \mathrm{cm}$ ). ${ }^{24}$ As shown in Figure 4, when applying an electric field along the $\mathrm{z}$ direction (normal to the inorganic plane), spin splitting is calculated for electrons in the $n=1$ single-layer slab, with a linear dependence on tilting angles; the obtained $\alpha \mathrm{R}$ value is nearly $0.8 \mathrm{eV} \cdot \AA$ for electrons at a field of $2.5 \times 10^{7} \mathrm{~V} / \mathrm{cm}$, but the effect is negligible for holes. The $n=2$ crystal retains its intrinsic band splitting feature when an electric field is applied, with the $\alpha \mathrm{R}$ values for electrons slightly increasing under very high electric fields (see Figure S6, Supporting Information).

\section{CONCLUSION}

We have confirmed the strong spin-orbit coupling and giant Rashba splitting in a prototype $3 \mathrm{D}$ perovskite $\mathrm{MAPbI}_{3}$ by combining the PBE functional with SOC effects and vdW interactions in our DFT calculations. We show that large Rashba coefficients for holes and electrons are also achieved in $2 \mathrm{D} \mathrm{MAPbI}_{3}$ structures with different terminations (0.5-1.0 eV $\AA \AA$ for Popper hybrid perovskites with $n=1,2$, and 3, we observed an intrinsic, large Rashba splitting only in (PEA) ${ }_{2} \mathrm{MAPb}_{2} \mathrm{I}_{7}(n=2)$, a feature which was experimentally proven by demonstrating the longer PL and ground-state bleaching recovery lifetimes present in this system. Our work highlights that controlling the number of layers in $2 \mathrm{D}$ RP perovskites must be considered in order to design stable low-dimensional hybrid perovskites with strong Rahsba effects for spintronic applications.

\section{ASSOCIATED CONTENT}

Supporting Information. Experimental and optimized crystal parameters; calculated Rashba coefficient; electronic band structures; X-ray diffraction pattern; and fitting parameters of TRPL spectra. This material is available free of charge via the Internet at http://pubs.acs.org.

\section{AUTHOR INFORMATION}

\section{Corresponding Author}

*E-mail: omar.abdelsaboor@kaust.edu.sa; jean-

luc.bredas@chemistry.gatech.edu.

\section{Notes}

The authors declare no competing financial interests.

\section{ACKNOWLEDGMENT}

This work was supported by the King Abdullah University of Science and Technology (KAUST) and by the Georgia Research Alliance and Vasser-Woolley Foundation through 
the chair in Molecular Design at the Georgia Institute of Technology. We acknowledge the Supercomputing Laboratory at KAUST for their computational and storage resources as well as their gracious assistance.

\section{REFERENCES}

(1) Stranks, S. D.; Snaith, H. J., Metal-Halide Perovskites for Photovoltaic and Light-Emitting Devices. Nat. Nanotechnol. 2015, 10, 391-402.

(2) Saliba, M.; Orlandi, S.; Matsui, T.; Aghazada, S.; Cavazzini, M.; Correa-Baena, J. P.; Gao, P.; Scopelliti, R.; Mosconi, E.; Dahmen, K. H., et al., A Molecularly Engineered HoleTransporting Material for Efficient Perovskite Solar Cells. Nat. Energy 2016, 1, 15017.

(3) Peng, W.; Wang, L. F.; Murali, B.; Ho, K. T.; Bera, A.; Cho, N.; Kang, C. F.; Burlakov, V. M.; Pan, J.; Sinatra, L., et al., SolutionGrown Monocrystalline Hybrid Perovskite Films for HoleTransporter-Free Solar Cells. Adv. Mater. 2016, 28, 3383-3390.

(4) Tsai, H. H.; Nie, W. Y.; Blancon, J. C.; Toumpos, C. C. S.; Asadpour, R.; Harutyunyan, B.; Neukirch, A. J.; Verduzco, R.; Crochet, J. J.; Tretiak, S., et al., High-Efficiency Two-Dimensional Ruddlesden-Popper Perovskite Solar Cells. Nature 2016, 536, 312316.

(5) Zhou, N.; Shen, Y.; Li, L.; Tan, S.; Liu, N.; Zheng, G.; Chen, Q.; Zhou, H., Exploration of Crystallization Kinetics in Quasi Two-Dimensional Perovskite and High Performance Solar Cells. J. Am. Chem. Soc. 2017, 140, 459-465.

(6) Wang, N. N.; Cheng, L.; Ge, R.; Zhang, S. T.; Miao, Y. F.; Zou, W.; Yi, C.; Sun, Y.; Cao, Y.; Yang, R., et al., Perovskite LightEmitting Diodes Based on Solution-Processed Self-Organized Multiple Quantum Wells. Nat. Photonics 2016, 10, 699-704.

(7) Chin, X. Y.; Cortecchia, D.; Yin, J.; Bruno, A.; Soci, C., Lead Iodide Perovskite Light-Emitting Field-Effect Transistor. Nat. Commun. 2015, 6, 7383 .

(8) Tsai, H.; Nie, W. Y.; Blancon, J. C.; Stoumpos, C. C.; Soe, C. M. M.; Yoo, J.; Crochet, J.; Tretiak, S.; Even, J.; Sadhanala, A., et al., Stable Light-Emitting Diodes Using Phase-Pure RuddlesdenPopper Layered Perovskites. Adv. Mater. 2018, 30, 1704217.

(9) Dou, L. T.; Yang, Y.; You, J. B.; Hong, Z. R.; Chang, W. H.; Li, G.; Yang, Y., Solution-Processed Hybrid Perovskite Photodetectors with High Detectivity. Nat. Commun. 2014, 5 , 5404 .

(10) Murali, B.; Saidaminov, M. I.; Abdelhady, A. L.; Peng, W.; Liu, J. K.; Pan, J.; Bakr, O. M.; Mohammed, O. F., Robust and AirStable Sandwiched Organo-Lead Halide Perovskites for Photodetector Applications. J. Mater. Chem. C 2016, 4, 2545-2552.

(11) Tian, W.; Zhou, H. P.; Li, L., Hybrid Organic-Inorganic Perovskite Photodetectors. Small 2017, 13, 1702107.

(12) Xing, G. C.; Mathews, N.; Lim, S. S.; Yantara, N.; Liu, X. F.; Sabba, D.; Gratzel, M.; Mhaisalkar, S.; Sum, T. C., LowTemperature Solution-Processed Wavelength-Tunable Perovskites for Lasing. Nat. Mater. 2014, 13, 476-48o.

(13) Birowosuto, M. D.; Cortecchia, D.; Drozdowski, W.; Brylew, K.; Lachmanski, W.; Bruno, A.; Soci, C., X-Ray Scintillation in Lead Halide Perovskite Crystals. Sci. Rep. 2o16, 6, 37254.

(14) Even, J.; Pedesseau, L.; Jancu, J. M.; Katan, C., Importance of Spin-Orbit Coupling in Hybrid Organic/Inorganic Perovskites for Photovoltaic Applications. J. Phys. Chem. Lett. 2013, 4, 29993005 .

(15) Kim, M.; Im, J.; Freeman, A. J.; Ihm, J.; Jin, H., Switchable $\mathrm{S}=1 / 2$ and $\mathrm{J}=1 / 2$ Rashba Bands in Ferroelectric Halide Perovskites. Proc. Natl. Acad. Sci. 2014, 111, 6900-6904.

(16) Kepenekian, M.; Even, J., Rashba and Dresselhaus Couplings in Halide Perovskites: Accomplishments and Opportunities for Spintronics and Spin-Orbitronics. J. Phys. Chem. Lett. 2017, 8, 3362-3370.
(17) Crepaldi, A.; Moreschini, L.; Autes, G.; Tournier-Colletta, C.; Moser, S.; Virk, N.; Berger, H.; Bugnon, P.; Chang, Y. J.; Kern, K., et al., Giant Ambipolar Rashba Effect in the Semiconductor Bitei. Phys. Rev. Lett. 2012, 109, 096803.

(18) Maass, H.; Bentmann, H.; Seibel, C.; Tusche, C.; Eremeev, S. V.; Peixoto, T. R. F.; Tereshchenko, O. E.; Kokh, K. A.; Chulkov, E. V.; Kirschner, J., et al., Spin-Texture Inversion in the Giant Rashba Semiconductor BiTeI. Nat. Commun. 2016, 7, 11621.

(19) Dresselhaus, G.; Kip, A. F.; Kittel, C., Spin-Orbit Interaction and the Effective Masses of Holes in Germanium. Phys. Rev. 1954, 95, 568-569.

(20) Rashba, E. I., Properties of Semiconductors with an Extremum Loop .1. Cyclotron and Combinational Resonance in a Magnetic Field Perpendicular to the Plane of the Loop. Sov. Phys. Solid State 1960, 2, 1109-1122.

(21) Bychkov, Y. A.; Rashba, E. I., Properties of a 2 D ElectronGas with Lifted Spectral Degeneracy. JETP Lett. 1984, 39, 78-81.

(22) Ishizaka, K.; Bahramy, M. S.; Murakawa, H.; Sakano, M.; Shimojima, T.; Sonobe, T.; Koizumi, K.; Shin, S.; Miyahara, H.; Kimura, A., et al., Giant Rashba-Type Spin Splitting in Bulk BiTeI. Nat. Mater. 2011, 10, 521-526.

(23) Odenthal, P.; Talmadge, W.; Gundlach, N.; Wang, R. Z.; Zhang, C.; Sun, D. L.; Yu, Z. G.; Vardeny, Z. V.; Li, Y. S., SpinPolarized Exciton Quantum Beating in Hybrid Organic-Inorganic Perovskites. Nat. Phys. 2017, 13, 894-899.

(24) Zhai, Y. X.; Baniya, S.; Zhang, C.; Li, J. W.; Haney, P.; Sheng, C. X.; Ehrenfreund, E.; Vardeny, Z. V., Giant Rashba Splitting in 2D Organic-Inorganic Halide Perovskites Measured by Transient Spectroscopies. Sci. Adv. 2017, 3, e1700704.

(25) Frohna, K.; Deshpande, T.; Harter, J.; Peng, W.; Barker, B. A.; Neaton, J. B.; Louie, S. G.; Bakr, O. M.; Hsieh, D.; Bernardi, M., Inversion Symmetry and Bulk Rashba Effect in Methylammonium Lead Iodide Perovskite Single Crystals. Nat. Commun. 2018, 9, 1829.

(26) Wang, J.; Pan, X.; Zhang, C.; Guo, H.; Vardeny, Z. V., LightControlled Spintronic Device Based on Hybrid Organic-Inorganic Perovskites. J. Photonics Energy 2018, 8, 032207.

(27) Hutter, E. M.; Gelvez-Rueda, M. C.; Osherov, A.; Bulovic, V.; Grozema, F. C.; Stranks, S. D.; Savenije, T. J., Direct-Indirect Character of the Bandgap in Methylammonium Lead Iodide Perovskite. Nat. Mater. 2017, 16, 115-120.

(28) Zhang, Y. H.; Yin, J.; Parida, M. R.; Ahmed, G. H.; Pan, J.; Bakr, O. M.; Bredas, J. L.; Mohammed, O. F., Direct-Indirect Nature of the Bandgap in Lead-Free Perovskite Nanocrystals. $J$. Phys. Chem. Lett. 2017, 8, 3173-3177.

(29) Yu, Z. G., The Rashba Effect and Indirect Electron-Hole Recombination in Hybrid Organic-Inorganic Perovskites. Phys. Chem. Chem. Phys. 2017, 19, 14907-14912.

(30) Zhang, X.; Shen, J. X.; Van de Walle, C. G., ThreeDimensional Spin Texture in Hybrid Perovskites and Its Impact on Optical Transitions. J. Phys. Chem. Lett. 2018, 9, 2903-2908.

(31) Alarousu, E.; El-Zohry, A. M.; Yin, J.; Zhumekenov, A. A.; Yang, C.; Alhabshi, E.; Gereige, I.; AlSaggaf, A.; Malko, A. V.; Bakr, O. M., et al., Ultralong Radiative States in Hybrid Perovskite Crystals: Compositions for Submillimeter Diffusion Lengths. J. Phys. Chem. Lett. 2017, 8, 4386-439o.

(32) Isarov, M.; Tan, L. Z.; Bodnarchuk, M. I.; Kovalenko, M. V.; Rappe, A. M.; Lifshitz, E., Rashba Effect in a Single Colloidal $\mathrm{CsPbBr}_{3}$ Perovskite Nanocrystal Detected by Magneto-Optical Measurements. Nano Lett. 2017, 17, 5020-5026.

(33) Becker, M. A.; Vaxenburg, R.; Nedelcu, G.; Sercel, P. C.; Shabaev, A.; Mehl, M. J.; Michopoulos, J. G.; Lambrakos, S. G.; Bernstein, N.; Lyons, J. L., et al., Bright Triplet Excitons in Caesium Lead Halide Perovskites. Nature 2018, 553, 189-193.

(34) Hsiao, Y. C.; Wu, T.; Li, M. X.; Hu, B., Magneto-Optical Studies on Spin-Dependent Charge Recombination and 
Dissociation in Perovskite Solar Cells. Adv. Mater. 2015, 27, 28992906.

(35) Niesner, D.; Wilhelm, M.; Levchuk, I.; Osvet, A.; Shrestha, S.; Batentschuk, M.; Brabec, C.; Fauster, T., Giant Rashba Splitting in $\mathrm{CH}_{3} \mathrm{NH}_{3} \mathrm{PbBr}_{3}$ Organic-Inorganic Perovskite. Phys. Rev. Lett. 2016, 117, 126401.

(36) Li, J. W.; Haney, P. M., Optical Spintronics in OrganicInorganic Perovskite Photovoltaics. Phys. Rev. B 2016, 93, 155432.

(37) Kepenekian, M.; Robles, R.; Katan, C.; Sapori, D.; Pedesseau, L.; Even, J., Rashba and Dresselhaus Effects in Hybrid Organic-Inorganic Perovskites: From Basics to Devices. ACS Nano 2015, 9, 11557-11567.

(38) Kresse, G.; Hafner, J., Ab-Initio Molecular-Dynamics for Open-Shell Transition-Metals. Phys. Rev. B 1993, 48, 13115-13118.

(39) Kresse, G.; Furthmuller, J., Efficient Iterative Schemes for Ab Initio Total-Energy Calculations Using a Plane-Wave Basis Set. Phys. Rev. B 1996, 54, 11169-11186.

(40) Peng, W.; Yin, J.; Ho, K. T.; Ouellette, O.; De Bastiani, M.; Murali, B.; El Tall, O.; Shen, C.; Miao, X. H.; Pan, J., et al., Ultralow Self-Doping in Two-Dimensional Hybrid Perovskite Single Crystals. Nano Lett. 2017, 17, 4759-4767.

(41) Kutes, Y.; Ye, L. H.; Zhou, Y. Y.; Pang, S. P.; Huey, B. D.; Padture, N. P., Direct Observation of Ferroelectric Domains in Solution-Processed Chznh3pbiz Perovskite Thin Films. J. Phys. Chem. Lett. 2014, 5, 3335-3339.

(42) Mosconi, E.; Etienne, T.; De Angelis, F., Rashba Band Splitting in Organohalide Lead Perovskites: Bulk and Surface Effects. J. Phys. Chem. Lett. 2017, 8, 2247-2252.

(43) Yin, J.; Yang, H.; Song, K.; El-Zohry, A. M.; Han, Y.; Bakr, O. M.; Brédas, J.-L.; Mohammed, O. F., Point Defects and Green
Emission in Zero-Dimensional Perovskites. J. Phys. Chem. Lett. 2018, 8, 5490-5495.

(44) Ast, C. R.; Henk, J.; Ernst, A.; Moreschini, L.; Falub, M. C.; Pacile, D.; Bruno, P.; Kern, K.; Grioni, M., Giant Spin Splitting through Surface Alloying. Phys. Rev. Lett. 2007, 98, 186807.

(45) Zhu, Z. H.; Levy, G.; Ludbrook, B.; Veenstra, C. N.; Rosen, J. A.; Comin, R.; Wong, D.; Dosanjh, P.; Ubaldini, A.; Syers, P., et al., Rashba Spin-Splitting Control at the Surface of the Topological Insulator $\mathrm{Bi}_{2} \mathrm{Se}_{3}$. Phys. Rev. Lett. 2011, 107, 186405.

(46) Ahmed, G. H.; Yin, J.; Bose, R.; Sinatra, L.; Alarousu, E.; Yengel, E.; AlYami, N. M.; Saidaminov, M. I.; Zhang, Y.; Hedhili, M. N., et al., Pyridine-Induced Dimensionality Change in Hybrid Perovskite Nanocrystals. Chem. Mater. 2017, 29, 4393-4400.

(47) Milot, R. L.; Sutton, R. J.; Eperon, G. E.; Haghighirad, A. A.; Hardigree, J. M.; Miranda, L.; Snaith, H. J.; Johnston, M. B.; Herz, L. M., Charge-Carrier Dynamics in $2 \mathrm{~d}$ Hybrid Metal-Halide Perovskites. Nano Lett. 2016, 16, 7001-7007.

(48) Zhang, Z. S.; Fang, W. H.; Tokina, M. V.; Long, R.; Prezhdo, O. V., Rapid Decoherence Suppresses Charge Recombination in Multi-Layer 2D Halide Perovskites: Time-Domain Ab Initio Analysis. Nano Lett. 2018, 18, 2459-2466.

(49) Eid, J.; Usman, A.; Gereige, I.; Van Duren, J.; Lyssenko, V.; Leo, K.; Mohammed, O. F., Ultrafast Pump-Probe Reflectance Spectroscopy: Why Sodium Makes $\mathrm{Cu}(\mathrm{In}, \mathrm{Ga}) \mathrm{Se}_{2}$ Solar Cells Better. Sol. Energy Mater. Sol. Cells 2015, 140, 33-37.

(5o) Leppert, L.; Reyes-Lillo, S. E.; Neaton, J. B., Electric Fieldand Strain-Induced Rashba Effect in Hybrid Halide Perovskites. J. Phys. Chem. Lett. 2016, 7, 3683-3689. 


\section{TOC graphic}

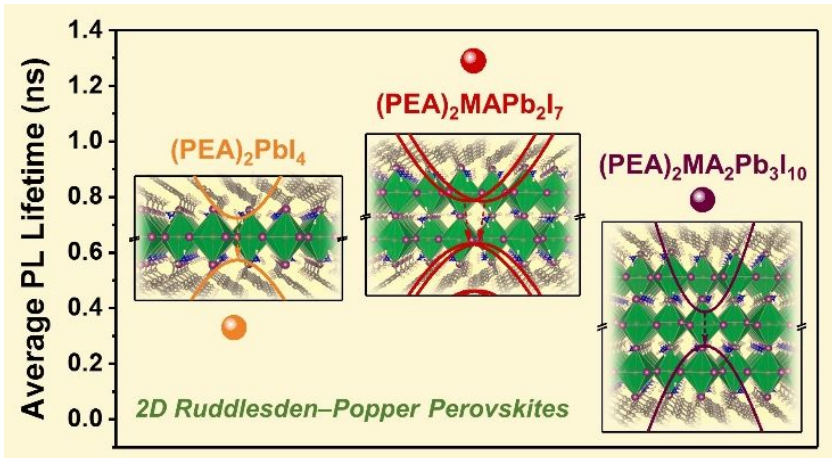

